\title{
Experimental of Vision-based Lane Markings Segmentation Methods in Lane Detection Application
}

\author{
Em Poh Ping ${ }^{1, *}, J$ Hossen ${ }^{1}$, Fitrian Imaduddin ${ }^{2}$, Wong Eng Kiong ${ }^{1}$ and Ubaidillah Sabino ${ }^{2}$ \\ ${ }^{1}$ Faculty of Engineering and Technology, Multimedia University, Jalan Ayer Keroh Lama, Bukit Beruang, 75450 Melaka, Malaysia \\ ${ }^{2}$ Faculty of Engineering, Universitas Sebelas Maret, Jalan Ir. Sutami 36A, Kentingan, Surakarta 57126, Central Java, Indonesia
}

Received 11 March 2019; Accepted 1 April 2019

\begin{abstract}
Lane departure collisions have contributed into the traffic accidents that cause millions of injuries and tens of thousands of casualties per year worldwide. Hence, a vision-based lane detection framework (VBLD) is proposed to detect lane markings on the road for unindented lane departure. The proposed VBLD framework is composed of colour space conversion, region of interest, lane marking segmentation, Hough transformation and peak detection, reverse Hough transformation, and draw detected lines on original image. Besides, finite impulse response saturation autothreshold (FIRSA) lane marking segmentation method is also proposed for lane edges extraction. For performance evaluation on the proposed VBLD framework and proposed FIRSA lane markings segmentation method, real-life datasets of road footages are collected using an instrumented vehicle. The outputs of lane detection frames from Clip \#1, \#2, \#3, and \#4 involving variety of road conditions are evaluated using detection rate, false positive rate, and false negative rate assessment metrics where the number of frames are manually counted using visual inspection. Experimental results have shown the evidences of the proposed VBLD framework using proposed FIRSA lane markings segmentation method obtained satisfactory lane detection results compared to benchmark lane marking segmentation methods.
\end{abstract}

Keywords: Lane Boundary Detection; Lane Markings Segmentation; False Positive Rate; False Negative Rate.

\section{Introduction}

Lane departure crashes count for the majority of highway fatalities and caused hundreds of human deaths, thousands of injuries, and billions of dollars in a loss every year. It is reported in [1], [2] that Malaysia had been ranked as the country with the highest fatality risk death per 100,000 population, in the world since 1996. Based on World Health Organisation (WHO) statistics for 2013 as per reported in [3],[4] that Malaysia was among the emerging countries with the hazardous roads after Thailand and South Africa. This statistical data agreed with the general road accident statistics in Malaysia for 2013 [5].

In global point of view, the regional distribution of 750,000 fatalities with almost half of all fatalities are coming from Asia in year 1999 [1], [2]. A similar trend of road fatalities as seen in year 1999, where the Asia continent holds more than half of all fatalities in year 2014 [6]. In between time period of 1999-2006, the worldwide average road traffic fatalities among motorised-four wheelers are estimated to be almost half of total fatalities at $45 \%$, followed by pedestrians, motorcyclists, and bicyclists user groups at $31 \%, 18 \%$, and $7 \%$, respectively [7]. A similar order of distributed road traffic deaths by road user type in year 2013 was found in [8], where the 4-wheeled vehicles attained $35 \%$, followed by pedestrians, motorized 2 - or 3 wheelers, cyclists and other road users at $31 \%, 22 \%, 11 \%$,

*E-mail address: ppem@mmu.edu.my ISSN: 1791-2377 @ 2019 Eastern Macedonia and Thrace Institute of Technology. All rights reserved. doi:10.25103/jestr.121.22 and $1 \%$, respectively. Many related studies found in [9]-[11] had shown that single vehicle lane departure crashes accounted largely in road traffic deaths that results from drifting out of the roadway into oncoming traffic, into adjacent traffic or off the roadway.

Furthermore, the road fatalities statistical data found in [1], [6] had shown an incline trend in worldwide traffic fatalities compared to the previous years, which agrees with the predicted future development of road fatalities in different regions of the world [12], [13]. Particularly in the region of South Asia, the predicted number of road fatalities in year 2020 is more than 3.5 times higher of the total road fatalities recorded in year 1990 .

In response to such stern problems, Lane Keeping Assistance (LKA) [14]; Lane Departure Warning (LDW) [15]-[17]; Lane Following (LF) [18], [19]; Lateral Control (LC); Intelligent Cruise Control (ICC); Collision Warning (CW) [20]; and Autonomous Vehicle Guidance [21] are called for by vehicle manufacturer industries to enhance the vehicle safety.

In general, research works on lane detection can be divided into two types. The first system is those without units of controllers, like LDW, Collision Warning (CW). This passive safety system only detects information and sends warning or reminding message if necessary. These systems are usually implemented in Driving Assistant System (DAS), instead of impacting on vehicles in autonomous vehicle guidance. The second type of system is designed with feedback namely active safety system, and with the aim of impacting on vehicle behaviour, which functions the way as a controller. In this paper, a passive type of lane detection framework is implemented. 
In this paper, a vision-based lane detection framework (VBLD) framework is proposed. The proposed VBLD framework is essentially consists of pre-processing stage and followed by detection stage. The pre-processing stage of VBLD framework is made up of colour space conversion, region-of-interest, and lane marking segmentation. The proposed finite impulse response saturation autothreshold (FIRSA) lane marking segmentation method used in this paper is represented by 2D-Finite impulse response (2D-FIR) filter followed by a saturation function and autothreshold. Whereas, the detection stage of VBLD framework is formed by Hough transformation and peak detection, reverse Hough transformation, and draw detected lines on original image. For the proposed VBLD framework performance evaluation, previous lane markings segmentation methods are used for comparative analysis.

The rest of this paper is organized as follows. Section II begins with the methodology of proposed VBLD framework, which contains pre-processing stage in subsection II-A and detection stage in subsection II-B. Section III contains the experimental results and discussion on the proposed VBLD framework. The experimental test bed is elaborated in subsection III-A, where the camera used in this paper is introduced in subsection III-B and description of real-life datasets in subsection III-C. The experimental results and discussion are detailed in subsection III-D. The paper is concluded and presented future work in section IV.

\section{Materials and Methods}

The VBLD framework is developed by using MATLAB Simulink software. Basically, the VBLD framework consists of two essential stages, which is pre-processing stage and followed by detection stage. Figure Fig. 1 shows an overall flow chart involving the proposed VBLD framework. In this section, the pre-processing stage of VBLD framework is introduced first in subsection II.A and followed by detection stage of VBLD framework in subsection II.B.

\subsection{Pre-processing Stage}

The pre-processing stage mainly consists of three main components, which is colour space conversion, a Region of Interest, and FIRSA lane marking segmentation method in the sequence order. The pre-processing stage depends on the input image (step 1. in Figure Fig. 1) from a Logitech C525 camera [22], which is fixed at the centre of front windscreen to capture the road footages [23], [24]. To simplify the problem, the camera is assumed that it is setup to make the baseline horizontal, which assures the horizon in the image (parallel to the $X$-axis of image plane). In this paper, it is assumed that the input image resolution to the preprocessing stage is a $320 \times 180$ Red, Green, Blue (RGB) colour image.

As an initial step, the input RGB colour image is converted into grayscale to obtain the grayscale image (step 2. in Figure Fig. 1). The captured colour image in RGB colour space is converted to grayscale colour space so that to reduce the processing time, less computational, and less sensitive to scene condition. Equation (1 represents the function which is to be applied on RGB image for converting the original image to grayscale image. The original image from frame 1 of Clip \#1 is shown in Figure Fig. 2, while Figure Fig. 3 shows the grayscale conversion of the original image.

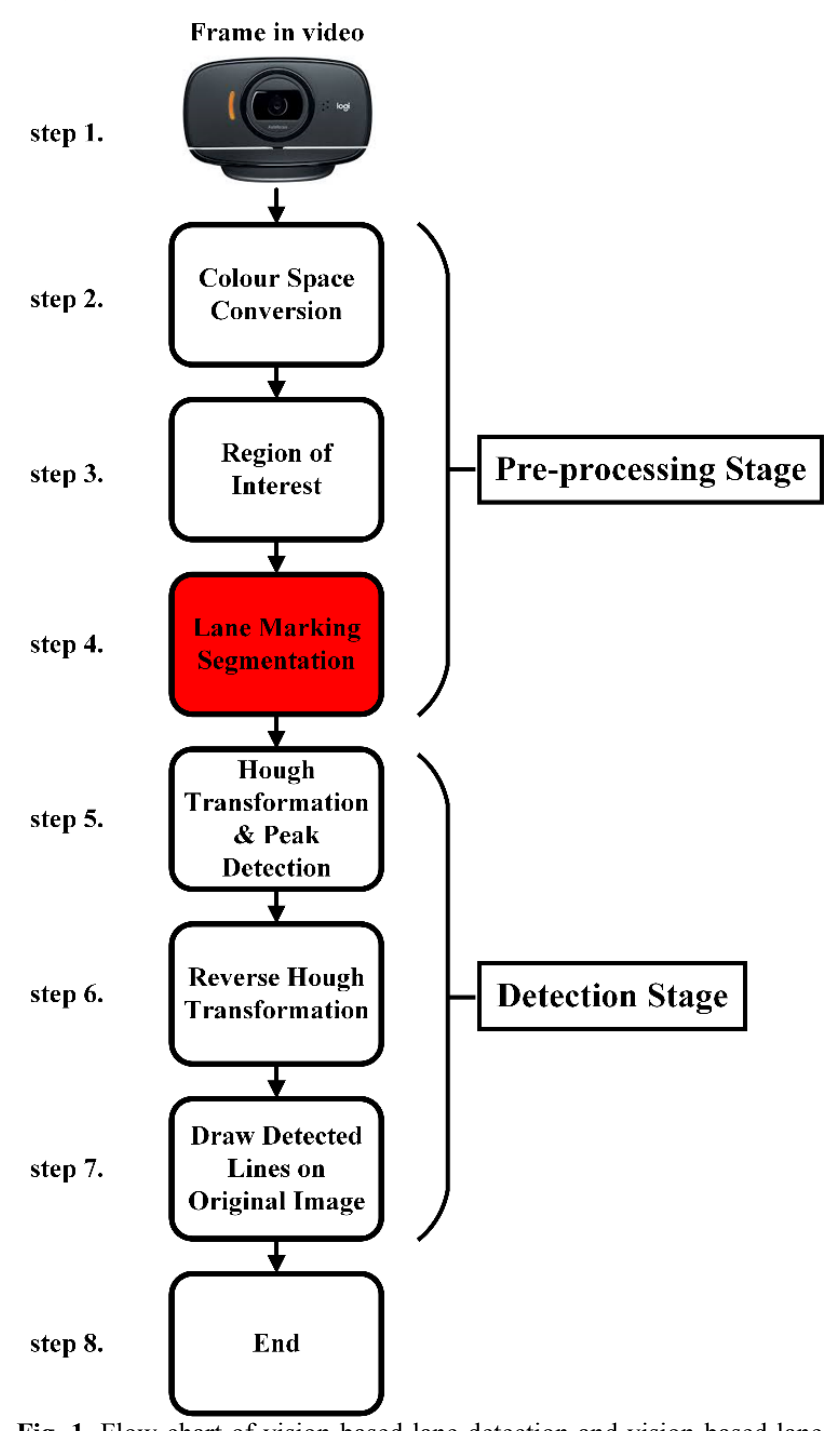

Fig. 1. Flow chart of vision-based lane detection and vision-based lane departure warning frameworks.

Grayscale $=\left[\begin{array}{lll}0.299 & 0.587 & 0.114\end{array}\right]\left[\begin{array}{c}R^{\prime} \\ G^{\prime} \\ B^{\prime}\end{array}\right]$

where

$R^{\prime}$ - Red component of the image

$G^{\prime}$ - Green component of the image

$B^{\prime}$ - Blue component of the image

The reason for employing a pre-processing stage is because the frame images in video taken in real world contain significant outliers other than actual lane markings. Images need to be properly prepared with outliers filtered out, depending on the requirements of different computer vision systems. It can be realized by finding a Region of Interest (ROI) and then followed by applying lane marking segmentation. Figure Fig. 6a shows the grayscale image after applying ROI onto the original frame image, and apparently the bottom half region of the image is selected as ROI [16] (step 3. in Figure Fig. 1). Commonly, the upper region of the frame image is considered as outliers due to unwanted features such as vehicles, road sign, and roadside trees. The image resolution of ROI image is reduced to $320 \times 90$.

The flow chart of FIRSA lane marking segmentation method as highlighted in red colour (step 4. in Figure Fig. 1) is mainly consist of three important blocks, which is a 2- 
Dimensional Finite Impulse Response (2-D FIR) filter block, followed by a Saturation block, and an Autothreshold block as illustrated in Figure Fig. 4. The FIRSA lane marking segmentation method is applied in step 4. of VBLD framework as shown in Figure Fig. 1. The 2-D FIR filter is a basic filter for image processing, which used to support in edge extraction [25] and noise removal [26]. Edge extraction is essential in image processing because edges represent a significant portion of characteristics contained in an image, for example the printed lane markings on road surface for lane detection application. In this paper, 2-D FIR filter [27] is used to extract the lane markings printed on the road surface for lane detection application. The 2-D FIR edge extraction is designed for the regions where light intensity changes slowly. It can differentiate the edge and to smooth the noise in a nosy image simultaneously. It is also computationally more efficient than the popular Laplacian of Gaussian method [26].

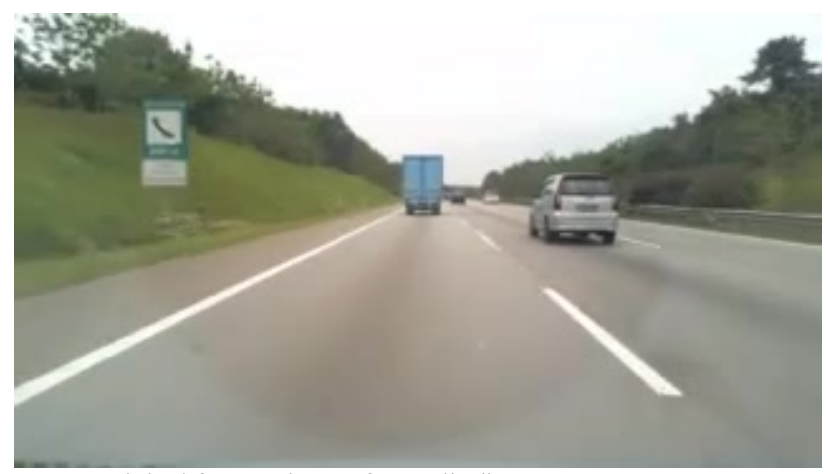

Fig. 2. Original frame 1 image from Clip \#1.

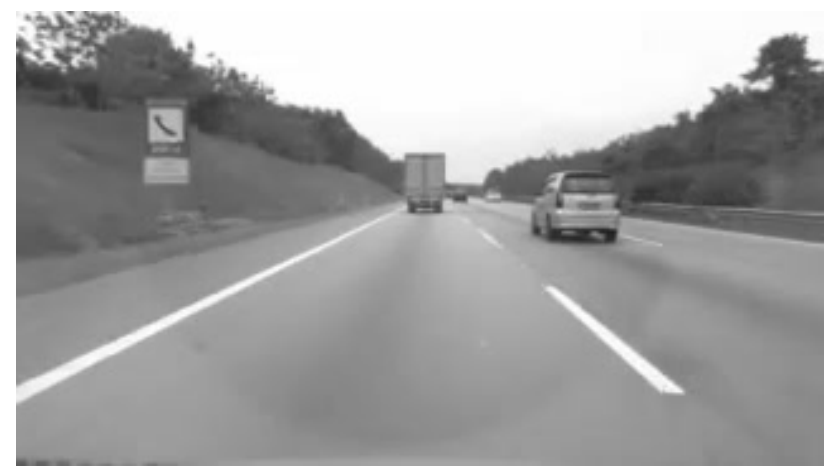

Fig. 3. RGB to gray-scale conversion of the original frame 1 image from Clip \#1.

In [27], 2-D FIR filtering operation in spatial convolution may be achieved using a neighbourhood averaging method, where the $H(i, j)$ pixel value is changed to the $C(i, j)$ pixel value as shown in Equation $\quad(2 . \quad$ In Equation $\quad(2, A$ denote the filter kernel and * denotes the convolution operation. The edge detection filter kernel in this case is $1 \times 3$ and each pixel in the filter kernel is as shown in Equation $A=\left[\begin{array}{ccc}-1 & 0 & 1\end{array}\right]$

(3.

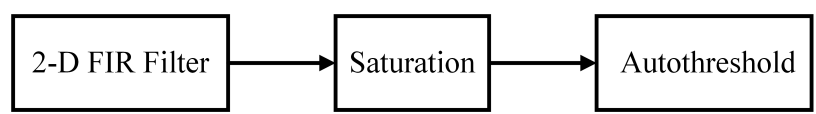

Fig. 4. Flow chart of Finite Impulse Response Saturation Autothreshold lane marking segmentation method as highlighted in red colour.

$$
\begin{aligned}
& C(i, j)=\sum_{m=0}^{(M a-1)} \sum_{n=0}^{(N a-1)} A(m, n) * H(i-m, j-n) \\
& A=\left[\begin{array}{lll}
-1 & 0 & 1
\end{array}\right]
\end{aligned}
$$

where $0 \leq i<M a+M h-1$ and $0 \leq j<N a+N h-1$ for full output size and replicate padding is applied for the pixels reference outside the image boundary. $C(i, j)$ is the filtered image, $H(i, j)$ is the ROI grayscale image, $A$ is the filter kernel, $m, n$ are the discrete spatial coordinates of the filter kernel, $\mathrm{Ma}, \mathrm{Na}$ are the original image dimensions, and $M h, N h$ are the filter kernel dimensions. Suppose that the filter kernel pixels are given as shown in the Figure Fig. 5 and every pixel of the filter kernel is considered by $-1 \leq m \leq 1$ only. Since the filter kernel in Equation $A=\left[\begin{array}{ccc}-1 & 0 & 1\end{array}\right]$ is a single row filter kernel, Equation ( 2 can be reduce to Equation (4.

$$
C(i, j)=\sum_{m=-1}^{m=1} A(m, n) * H(i-m, j)
$$

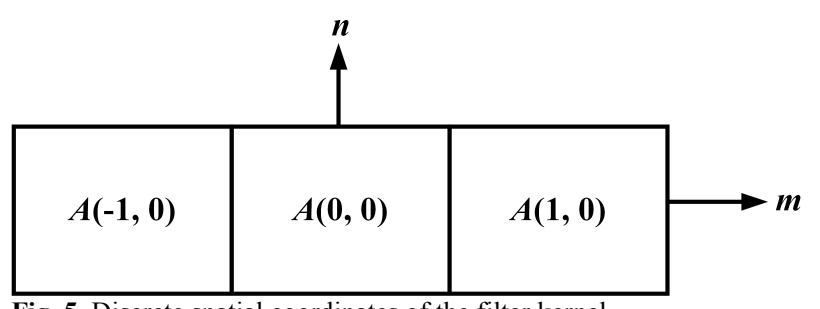

Fig. 5. Discrete spatial coordinates of the filter kernel.

Figures Fig. 6a-Fig. 6b show the input ROI grayscale image and the surface plot of input ROI grayscale image for frame 1 image of Clip \#1, respectively. The irregularity is observed in the input ROI grayscale image surface plot as shown in Figure Fig. $6 \mathrm{~b}$ due to unwanted noises appear on the road surface and side road. Still, the lane markings appeared as white pixels in Figure Fig. 6a are able translated as the peaks in $Z$-axis of surface plot in Figure Fig. 6b. In order to remove unwanted noise pixels appeared in the input ROI grayscale image and distinctly extract the lane markings from the input ROI grayscale image, 2-D FIR filter is then applied onto the input ROI grayscale image. Figures Fig. 6cFig. 6d show the filtered image and the surface plot of filtered image for frame 1 image of Clip \#1, respectively. From the filtered image and surface plot of filter image, it is noticed that the lane markings white pixels in Figure Fig. 6c are clearly stand out from the rest of the image pixels, which translated into distinct peaks with flat reference surface at zero intensity in Figure Fig. 6d. Still, non-positive distinct peaks are observed in the surface plot of filtered image in Figure Fig. 6d due to [ $\left.\begin{array}{ccc}-1 & 0 & 1\end{array}\right]$ filter kernel used in 2-D FIR. Hence, in order to remove non-positive distinct peaks appeared in Figure Fig. 6d, a saturation is applied to the filtered image as shown in the flow chart of FIRSA lane marking segmentation method in Figure Fig. 4.

The output signal of 2-D FIR is connected to a saturation block as shown in Figure Fig. 4, which produces an output signal that is the value of the input signal bounded to the upper saturation value of (1) and lower saturation value of $(0)$. The specified upper and lower limits are used to saturate the unwanted range of signals $(C(i, j)<0)$. Figures Fig. 6e- 
Fig. 6f show the saturated image and the surface plot of saturated image for frame 1 image of Clip \#1, respectively. It is observed that the non-positive distinct peaks have been saturated to a reference intensity value of $(0)$. The saturated signals are then connected to the autothreshold block as shown in Figure Fig. 4.

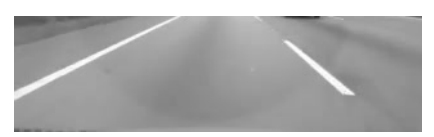

(a) Input Region on Interest grayscale image

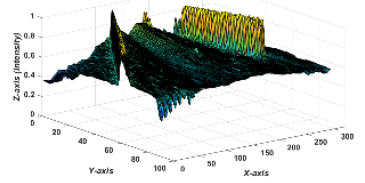

(b) Surface plot of grayscale image

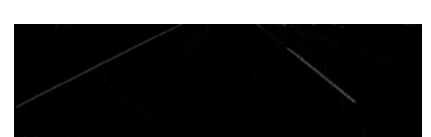

(c) Filtered image

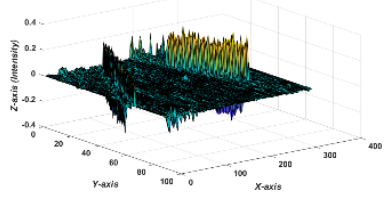

(d) Surface plot of filtered image

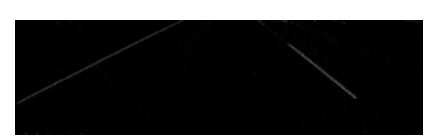

(e) Saturated image

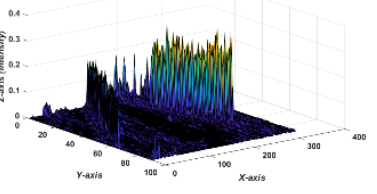

(f) Surface plot of saturated image

Fig. 6. Images (a), (c), and (e) represent the input Region of Interest grayscale image, filtered image, and saturated image for frame 1 of Clip \#1 original image, respectively. Images (b), (d), and (f) represent the surface plot of grayscale image, surface plot of filtered image, and surface plot of saturated image for the frame 1 of Clip \#1 original image, respectively.

Autothreshold block in the flow chart of FIRSA lane marking segmentation method as shown in Figure Fig. 4 is a binarization step, which required to highlight the lane marking of the filtering images. The core problem of binarization is how to determine the optimal threshold; if the threshold is excessively large, then a lane edge point will be missed or some redundant information will be detected. The FIRSA lane marking segmentation method employed Otsu thresholding method to perform binarization [28].

In this Otsu thresholding method, the histogram of image pixel values is examined for choosing a threshold automatically. The idea of this Otsu thresholding method is to search for two peaks, one representing foreground pixel value and one representing background pixel value, and choose a point in between the two peaks as the threshold value. The frame 1 of Clip \#1 filtering image is chosen and applied Otsu thresholding method to get a normalised threshold value. Then, the normalized threshold value (highlighted in red solid line) is plotted together with the histogram for the frame 1 of Clip \#1 filtering image as shown in Figure Fig. 7. The thresholded image based on Otsu thresholding method for frame 1 of Clip \#1 filtering image is as shown in Figure Fig. 8.

The threshold selection work is based on entirely on the set of histogram counts. To show the Otsu thresholding computation, pixels can take on the set of values $i=1,2, \ldots, L$, where $L$ represents the gray levels of the pixels for frame 1 of Clip \#1 filtering image. The histogram count for pixel value $i$ is $n_{i}$, and the associated probability is $p_{i}=n_{i} / N$, where $N$ is the number of image pixels. The thresholding task is formulated as the problem of dividing image pixels into two classes. $C_{0}$ is the set of pixels with values $[1, \ldots, k]$, and $C_{1}$ is the set of pixels with values in the range $[k+1, \ldots, L]$, where $k$ is the level of threshold value. The overall class probabilities, $\omega_{0}$ and $\omega_{1}$, are shown in Equations (5 and (6, respectively.

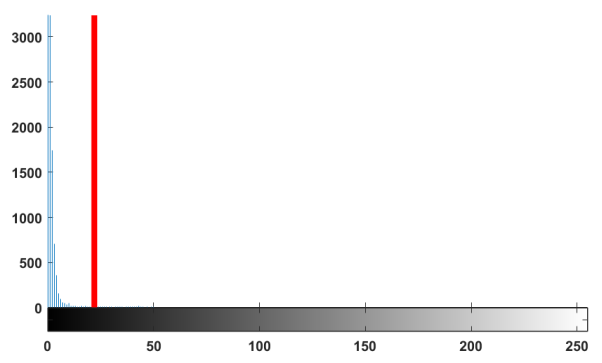

Fig. 7. Histogram and a normalized threshold (highlighted in red solid line) for the frame 1 of Clip \#1 filtering image.

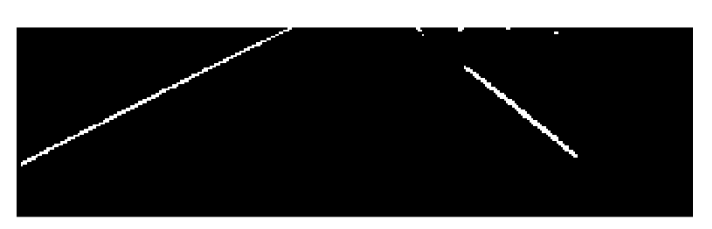

Fig. 8. Thresholded image for the frame 1 of Clip \#1 filtering image.

$$
\begin{aligned}
& \omega_{0}=\sum_{i=1}^{k} p_{i}=\omega(k) \\
& \omega_{1}=\sum_{i=k+1}^{L} p_{i}=1-\omega_{0}(k)
\end{aligned}
$$

The class means, $\mu_{0}$ and $\mu_{1}$, are the mean values of the pixels in $C_{0}$ and $C_{1}$. They are given by Equations ( 7 and (8 [29]-[32],

$$
\begin{aligned}
& \mu_{0}=\sum_{i=1}^{k} i p_{i} / \omega_{0}=\mu(k) / \omega(k) \\
& \mu_{1}=\sum_{i=k+1}^{L} i p_{i} / \omega_{1}=\frac{\mu_{T}-\mu(k)}{1-\omega(k)}
\end{aligned}
$$

where

$$
\mu(k)=\sum_{i=1}^{k} i p_{i}
$$

and $\mu_{T}$, the mean pixel value for the total image is shown in Equation

$$
\mu_{T}=\sum_{i=1}^{L} i p_{i}
$$


The class variances, $\sigma_{0}^{2}$ and $\sigma_{1}^{2}$, are shown in Equations (11 and

(12, respectively.

$\sigma_{0}^{2}=\sum_{i=1}^{k}\left(i-\mu_{0}\right)^{2} p_{i} / \omega_{0}$

$\sigma_{1}^{2}=\sum_{i=k+1}^{L}\left(i-\mu_{1}\right)^{2} p_{i} / \omega_{1}$

[28] mentioned three measures of goodness class separability namely within-class variance $(\lambda)$, betweenclass variance $(\kappa)$, and total variance $(\eta)$. These are given by Equations $(13,(14$, and $(15$, respectively,

$$
\begin{aligned}
& \lambda=\sigma_{B}^{2} \\
& \kappa=\sigma_{T}^{2} / \sigma_{W}^{2} \\
& \eta=\sigma_{B}^{2} / \sigma_{T}^{2}
\end{aligned}
$$

where

$$
\begin{aligned}
& \sigma_{W}^{2}=\omega_{0} \sigma_{0}^{2}+\omega_{1} \sigma_{1}^{2} \\
& \sigma_{B}^{2}=\omega_{0}\left(\mu_{0}-\mu_{T}\right)^{2}+\omega_{1}\left(\mu_{1}-\mu_{T}\right)^{2}=\omega_{0} \omega_{1}\left(\mu_{1}-\mu_{0}\right)^{2}
\end{aligned}
$$

[28] went on to point out that maximizing any of these criteria is equivalent to maximizing the others. Further, maximizing $\eta$ is the same as maximizing $\sigma_{B}^{2}$, which can be rewritten in terms of the selected threshold, $k$ as shown in Equation

$$
\sigma_{B}^{2}(k)=\frac{\left[\mu_{T} \omega(k)-\mu(k)\right]^{2}}{\omega(k)[1-\omega(k)]}
$$

The Equation

(18 is the essence of the algorithm. $\sigma_{B}^{2}$ is computed for all possible threshold values, and the threshold value that maximises $\sigma_{B}^{2}$ is chosen. Figure Fig. 9 illustrated the plot of histogram and $\sigma_{B}^{2}$ with the Otsu thresholding method choosing the place, where $\sigma_{B}^{2}$ is the highest as the threshold (highlighted in red colour dashed line) for frame 1 of Clip \#1 filtering image.

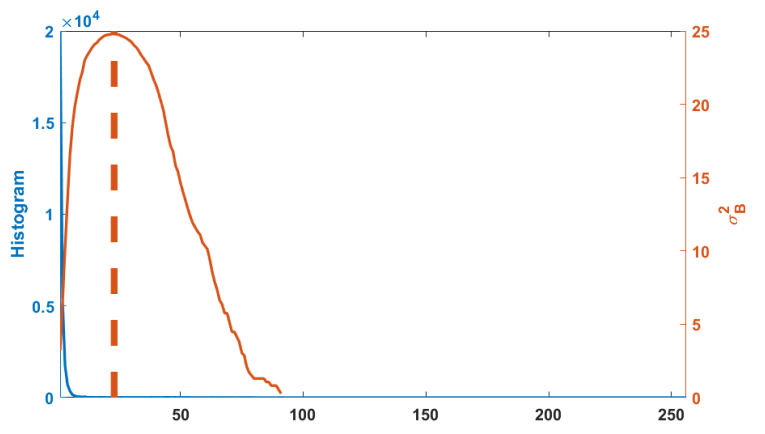

Fig. 9. Plot of histogram and $\sigma_{B}^{2}$ with the peak of $\sigma_{B}^{2}$ chosen as the threshold for the frame 1 of Clip \#1 filtering image.

\subsection{Detection Stage}

The Hough transform (HT) [33] is a technique that recognizes specific configuration of points in an image, such as lane segments, curves, or other patterns. The fundamental principle is that the form sought may be expressed via a known function depending on a set of parameters. A particular instance of the form sought is therefore entirely specified by the values taken by such a set of parameters.

For example, by taking as a representation of straight lanes, the Equation (19, any straight lane is entirely specified by the value of the parameters $(a, b)$. Equivalently, if one takes a different type of representation, as in Equation pair $(\rho, \theta)$.

(20, the straight lane is completely specified by the

$Y=a X+b$

$\rho=X \cos (\theta)+Y \sin (\theta)$

To illustrate on how the detection stage operated, the edge detection is performed on the frame 1 image of Clip \#1 as shown in Figure Fig. 8 so that to find the left and right lane edges of the segmented lane markings in the binary image. In this paper, a standard Hough transformation is used to perform edge detection to the lane markings (step 5 . in Figure Fig. 1). Figure Fig. 10 shows the result of Hough transformation in parameter plane for the frame 1 of Clip \#1 image with theta, $\theta$, resolution configured to $\pi / 180$ radians and rho, $\rho$, resolution is configured to 1 . The range of theta, $\theta$, is configured within the range of -1.2217 radians to 1.1868 radians. Local maxima detection is applied onto the parameter plane to extract the peaks that correspond to the left and right lane boundaries (step 5. in Figure Fig. 1). Figure Fig. 10 shows the results of local maxima detection with the highlighted two red squares to indicate right and left lane boundaries at $(-0.8901,120)$ and $(1.1,58)$, respectively. The local maxima is configured with two maxima number of local maxima, neighbourhood size at [319 89], and threshold equals to 1 . 


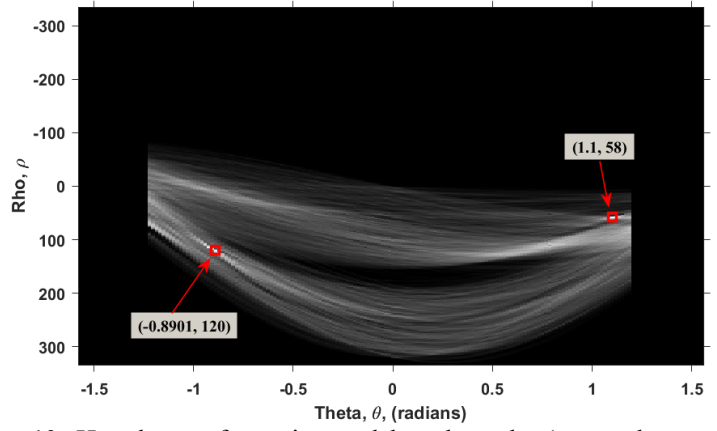

Fig. 10. Hough transformation and hough peaks (two red squares) in parameter plane for the frame 1 of Clip \#1 image.

A reserve Hough transformation is carried out on the hough peaks in order to display the detected lane boundaries for the frame 1 of Clip \#1 image (step 6. in Figure Fig. 1). A reserve Hough transformation's step involved the identification of the Cartesian coordinates of intersection between the reference image boundary lines and the lines described by $(\rho, \theta)$ pairs. Figure Fig. 11 shows the example of Line 1 intersecting the boundaries of the reference image at $\left[\left(X_{11}, Y_{11}\right)\left(X_{12}, Y_{12}\right)\right]$ and Line 2 interesting the boundaries at $\left[\left(X_{21}, Y_{21}\right)\left(X_{22}, Y_{22}\right)\right]$. The Cartesian coordinates of $\left(X_{11}, Y_{11}\right)$, $\left(X_{12}, Y_{12}\right),\left(X_{21}, Y_{21}\right)$, and $\left(X_{22}, Y_{22}\right)$ can be calculated from the Equation 20. Whereas, Figure Fig. 12 shows the results of reverse Hough transformation with the ROI grayscale image for frame 1 image of Clip \#1 as the ground truth. The image boundary intersection points are highlighted using black colour " $X$-mark" marker with the black colour solid lines represent Hough lines as shown in Figure Fig. 12. Both of the Hough lines are inline with the ground truth left and right lane boundaries. The $(-0.8901,120)$ Hough peak indicates left lane boundary and $(1.1,58)$ Hough peak indicates right lane boundary.

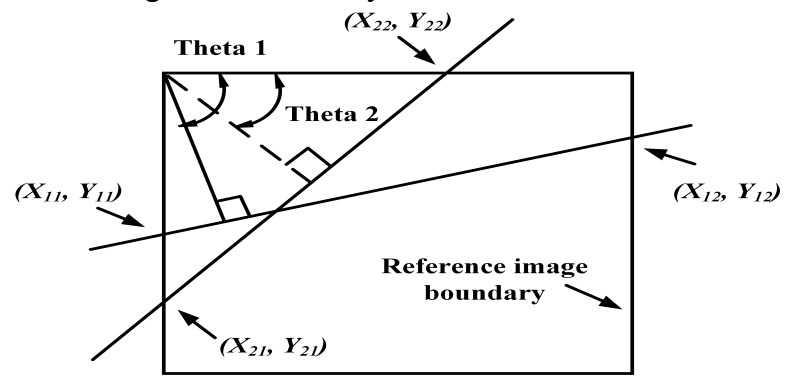

Fig. 11. Example illustration of Hough lines intersection.

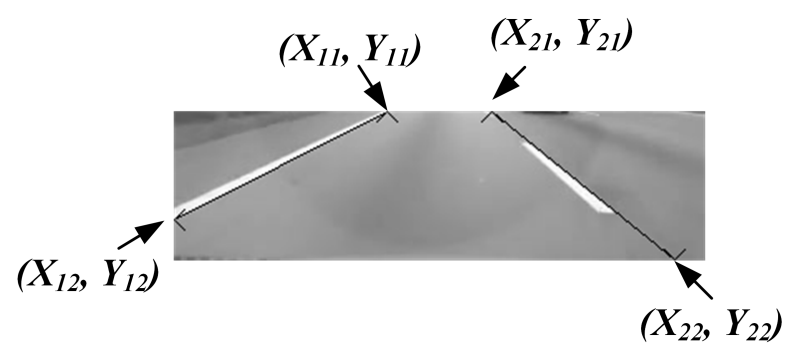

Fig. 12. Hough lines on the Region of Interest grayscale image for frame 1 of Clip \#1.

The Cartesian coordinates for $\left(Y_{11}\right),\left(Y_{12}\right),\left(Y_{21}\right)$, and $\left(Y_{22}\right)$ are required to be extended for another 90 pixels vertically due to the selection of bottom half of image as
ROI. The Cartesian coordinates for $\left(X_{11}, Y_{11}\right)$ and $\left(X_{12}, Y_{12}\right)$ are linked with an overlay red line that correspond to the left lane boundary (step 7. in Figure Fig. 1). Whereas, the Cartesian coordinates for $\left(X_{21}, Y_{21}\right)$ and $\left(X_{22}, Y_{22}\right)$ are linked with an overlay red line that correspond to the right lane boundary (step 7. in Figure Fig. 1). Both overlaid red lines show correct lane detection in original image for frame 1 of Clip \#1 as shown in Figure 13.

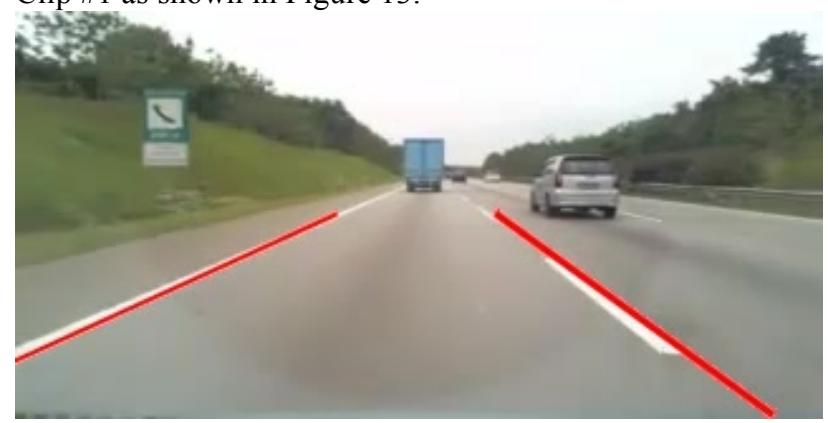

Fig. 13.Correct lane detection for the frame 1 image of Clip \#1.

\section{Results and discussion}

This section is organized into 4 subsections, which covers the experimental test bed, camera setup, description of reallife datasets, and experimental results and discussion in sequence order. Emphasis will be laid in subsection III-D for presenting the lane detection performance evaluation on the proposed FIRSA and benchmark lane marking segmentation methods using the proposed VBLD framework.

\subsection{Experimental Test Bed}

The proposed VBLD framework is implemented with MATLAB and Simulink, under the operating system of Windows 10, using Intel core $i 58250 \mathrm{U}$ processor and $4 \mathrm{~GB}$ RAM. Figure Fig. 14 shows how the video clip footages are acquired off-line so that trimming can be done on the video clip footages before running the proposed VBLD simulation. The experimental test bed setup used for the experimentation is shown in Figure Fig. 15. The instrumented vehicle used in the experimentation is Perodua Kancil 660 EX [34].

\subsection{Camera Setup}

The video sensing device that used to collect video footages is a Logitech C525 [22]. The camera is attached to the front windscreen at a height of $1.045 \mathrm{~m}$ above the ground and located at the centre of windscreen as shown in Figure Fig. 16. The frame rate of the video is 30 frame per second (FPS). Since the frame size has an enormous impact on the total speed of the system, the images resolution are reduced to $320 \times 180$ after being captured from camera, for real-time image processing.

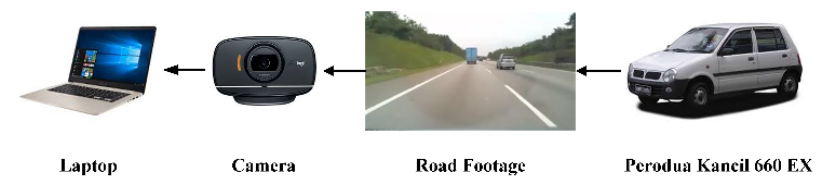

Fig. 14. Experimental test bed for acquiring real-life datasets for the vision-based lane detection framework. 
Em Poh Ping, J. Hossen, Fitrian Imaduddin, Wong Eng Kiong and Ubaidillah Sabino/

Journal of Engineering Science and Technology Review 12 (1) (2019) 185 - 195

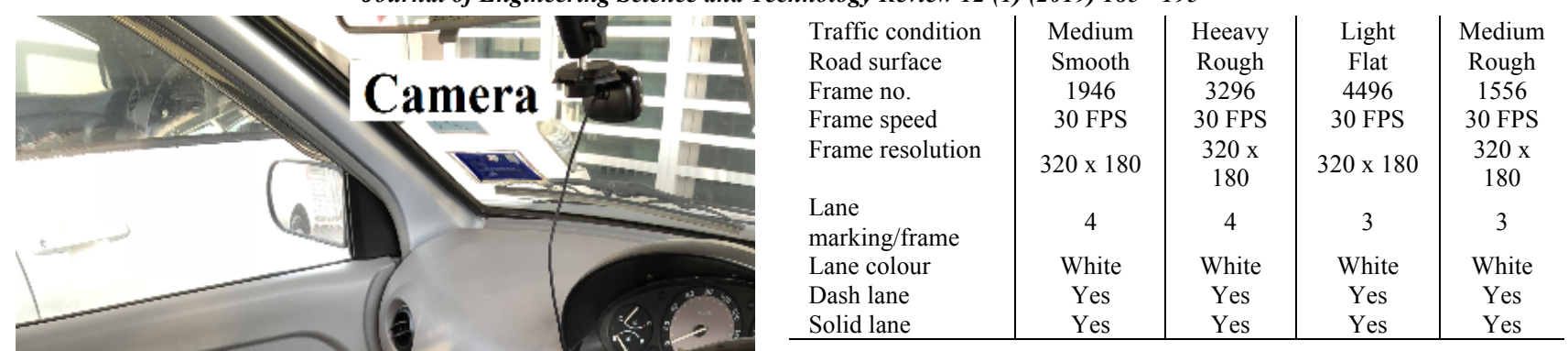

\subsection{Experimental Results and Discussion}

In this subsection, tabularly comparative experiments are performed to evaluate the efficiency and accuracy of the proposed FIRSA lane marking segmentation method using the proposed VBLD framework.

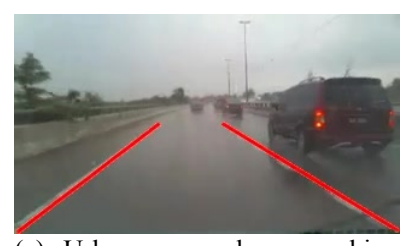

(a) Urban area: lane marking occluded with vehicles, heavy rain

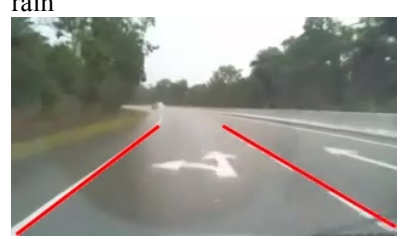

(c) Urban area: printed road-sign with curvy lane markings, light rain

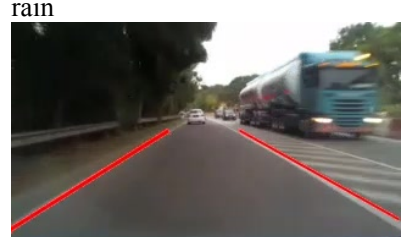

(e) Urban area: heavy traffic with multiple lane markings, cloudy weather

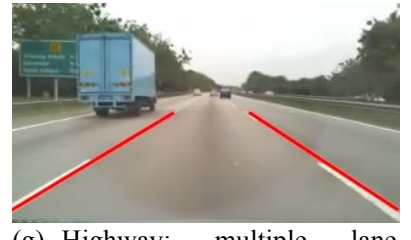

(g) Highway: multiple lane markings with medium traffic, sunny

Fig. 17. Correct lane detection using the proposed FIRSA lane marking segmentation method in the proposed VBLD framework.

Figure Fig. 17 shows some frame images with correct detection of lane markings. These pictures show that the proposed FIRSA lane marking segmentation method performs well in variety of real world scenarios. The proposed FIRSA and benchmark lane marking segmentation methods are implemented and included in Tables Table 2. I,

In Clip \#1, the proposed FIRSA lane marking segmentation method increases the detection rate from 43.63 $\%$ of Canny method to $96.45 \%$, which dramatically decreases the FPR from $54.73 \%$ to $3.44 \%$. Furthermore, the proposed FIRSA lane marking segmentation method decreases FPR and FNR compared to the benchmark lane marking segmentation methods (Canny, Sobel, Prewitt, and Roberts).

Table 1. Real-life Datasets of Acquired Road Footages

\begin{tabular}{l|c|c|c|c}
\hline Parameters & Clip \#1 & Clip \#2 & Clip \#3 & Clip \#4 \\
\hline Weather & Sunny & Cloudy & Light rain & $\begin{array}{c}\text { Heavy } \\
\text { rain } \\
\text { Location }\end{array}$ \\
Highway & Urban & Urban & Urban
\end{tabular}


$\mathrm{T}, \mathrm{T}$, and Table for comparative performance evaluation. In benchmark lane markings segmentation methods, Canny method uses lane marking segmentation founded on the Canny algorithm [35], followed by Hough transformation to detect lane markings. The Sobel method uses lane marking segmentation founded on Sobel algorithm [36], followed by Hough transformation to detect lane markings. The Prewitt method uses lane marking segmentation founded on Prewitt algorithm [37], followed by Hough transformation to detect lane markings. Whereas the Roberts method uses lane marking segmentation founded on Roberts algorithm [38], followed by Hough transformation to detect lane markings. The reason behind this choice is to show the precision and effectiveness of the proposed FIRSA lane marking segmentation method against benchmark lane marking segmentation methods. Admitting the drawbacks of the proposed FIRSA lane marking segmentation method, some images with lane detection failure have been illustrated in Figure Fig. 18, also served as a demonstration about the false positive and false negative results.

The evaluation of each lane marking segmentation method is performed by using some performance assessment metrics. The total number of all frame images formula is given in Equation 21. The formula used to calculate detection rate (DR) metric is shown in Equation 22. Two additional metrics are used, which are false positive rate (FPR) and false negative rate (FNR). False positive and false negative are considered the presence or absence of lane markings, respectively. FPR refers to the probability of falsely selecting a given object or contours such as vehicles, curbs of road, trees or electric poles, as a lane marking. The formula used to calculate FPR is shown in Equation 23. On the other hand, FNR refers to the situation when a lane marking is falsely rejected by the lane detection scheme. The formula used to calculate FNR is shown in Equation 24.

$$
\begin{aligned}
& N_{\text {Total }}=N_{D R}+N_{F P R}+N_{F N R} \\
& D R=\frac{N_{D R}}{N_{\text {Total }}} \times 100 \% \\
& F P R=\frac{N_{F P R}}{N_{\text {Total }}} \times 100 \% \\
& F N R=\frac{N_{F N R}}{N_{\text {Total }}} \times 100 \%
\end{aligned}
$$

where

$$
\begin{aligned}
& N_{\text {Total }} \text { - Total number of all frame images } \\
& N_{D R} \text { - Total number of correctly detected frame images } \\
& N_{F P R} \text { - Total number of false positive frame images } \\
& N_{F N R} \text { - Total number of false negative frame images }
\end{aligned}
$$

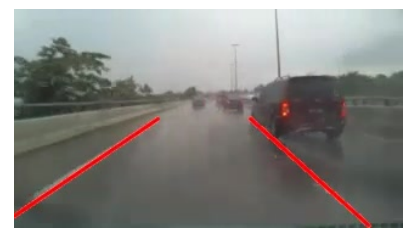

(a)

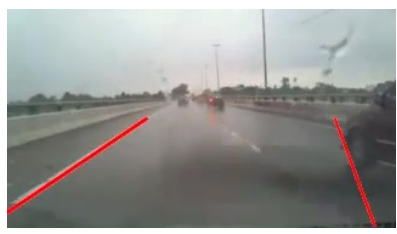

(b)

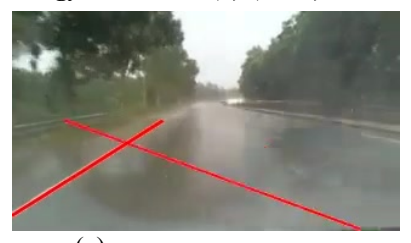

(c)

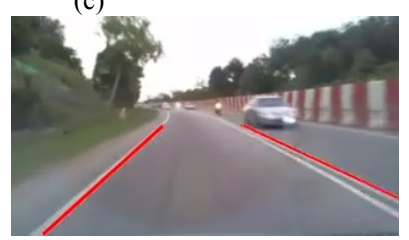

(e)

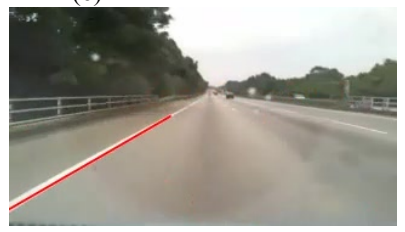

(g)

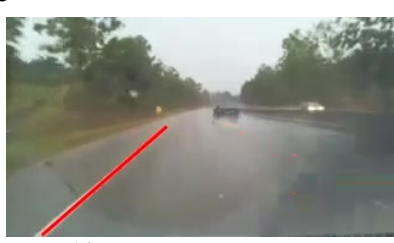

(d)

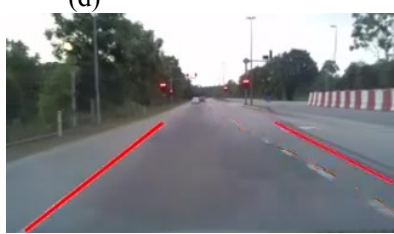

(f)

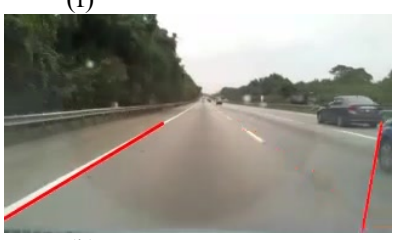

(h)
Fig. 18. Example of false positive and false negative results of the proposed FIRSA lane marking segmentation method: images (a) and (e) show the false positive results as well as correct detection; images (b), (c), (f), (h) show the false positive results for one lane marking while the other one being correctly detected; images $(\mathrm{d})$ and $(\mathrm{g})$ show the false negative results for right lane marking while the left lane marking is being correctly detected.

Different experimental results are presented in Tables 2, 3 , 4, and 5 for sunny, cloudy, light rain, and heavy rain weather, respectively. Table 2shows that the proposed FIRSA lane marking segmentation method performs better than the benchmark lane marking segmentation methods for Clip \#1, which represents a common situations in the highway.

Table 2. IComparison of lane marking segmentation methods for Clip \#1

\begin{tabular}{l|l|l|l}
\hline Methods & $\begin{array}{l}\text { Detection } \\
\text { Rate }\end{array}$ & $\begin{array}{l}\text { False } \\
\text { Positive } \\
\text { Rate }\end{array}$ & $\begin{array}{l}\text { False } \\
\text { Negative } \\
\text { Rate }\end{array}$ \\
\hline Proposed & $96.45 \%$ & $3.44 \%$ & $0.10 \%$ \\
FIRSA & $43.63 \%$ & $54.73 \%$ & $1.64 \%$ \\
Canny & $74.20 \%$ & $25.64 \%$ & $0.15 \%$ \\
Sobel & $72.61 \%$ & $27.29 \%$ & $0.10 \%$ \\
Prewitt & $76.57 \%$ & $23.07 \%$ & $0.36 \%$ \\
Roberts & \multicolumn{3}{|l}{} \\
\hline
\end{tabular}

In Clip \#1, the proposed FIRSA lane marking segmentation method increases the detection rate from 43.63 $\%$ of Canny method to $96.45 \%$, which dramatically decreases the FPR from $54.73 \%$ to $3.44 \%$. Furthermore, the proposed FIRSA lane marking segmentation method decreases FPR and FNR compared to the benchmark lane marking segmentation methods (Canny, Sobel, Prewitt, and Roberts).

Table 3. Comparison of lane marking segmentation methods for Clip \#2

\begin{tabular}{l|l|l|l}
\hline Methods & $\begin{array}{l}\text { Detection } \\
\text { Rate }\end{array}$ & $\begin{array}{l}\text { False } \\
\text { Positive } \\
\text { Rate }\end{array}$ & $\begin{array}{l}\text { False } \\
\text { Negative } \\
\text { Rate }\end{array}$ \\
\hline $\begin{array}{l}\text { Proposed } \\
\text { FIRSA }\end{array}$ & $86.17 \%$ & $13.83 \%$ & $0.00 \%$ \\
Canny & $64.08 \%$ & $35.62 \%$ & $0.30 \%$
\end{tabular}




\begin{tabular}{l|l|l|l} 
Sobel & $90.66 \%$ & $9.34 \%$ & $0.00 \%$ \\
Prewitt & $89.96 \%$ & $10.04 \%$ & $0.00 \%$ \\
Roberts & $91.66 \%$ & $8.34 \%$ & $0.00 \%$ \\
\hline
\end{tabular}

It is shown in Table 3, in spite of highest detection rate and false positive rate in Clip \#1, the proposed FIRSA lane marking segmentation method possesses some instability when it comes to Clip \#2, which having detection rate of $86.17 \%$ and false positive rate of $13.83 \%$. The proposed FIRSA lane marking segmentation method encountered slightly higher false positive rate mainly due to the incorrect lane marking detection, particularly the one on the right lane marking as illustrated in Figure Fig. 18e. Instead of detecting the right lane marking for driving direction, the proposed FIRSA lane marking segmentation method detected the right lane marking for oncoming driving direction as well, hence, the false positive detection result. Benchmark lane marking segmentation methods have much higher detection rate (91.66 \% for Roberts method, $90.66 \%$ for Sobel method, and $89.96 \%$ for Prewitt method) and lower fals positive rate (only $8.34 \%$ for Roberts method, $9.34 \%$ for Sobel method, and $10.04 \%$ for Prewitt method) except Canny method. This fact is mainly because poor illumination during cloudy weather makes the noisy edges less visible. It is also worth to take note that the Canny method performs adequately better in Clip \#2 compared to Clip \#1, \#3, and \#4, in spite of lower detection rate, and higher false positive rate and false negative rate in most cases.

Table $\mathrm{T}$ shows that the proposed FIRSA lane marking segmentation method in Clip \#3 achieved highest detection rate of $97.53 \%$ and lowest false positive rate of $2.45 \%$. The proposed FIRSA lane marking segmentation method increases the detection rate by $0.82 \%, 1.67 \%, 1.78 \%$, and $65.59 \%$, compared to Roberts, Sobel, Prewitt, and Canny methods, respectively. To eliminate the instability of Canny method, the proposed FIRSA lane marking segmentation method can be used to enhance the false positive rate result effectively. Besides false positive rate, the proposed FIRSA lane marking segmentation method performs significantly better than the benchmarks lane marking segmentation methods during light rain weather condition. This phenomenon can be related to the evidence on the proposed FIRSA lane marking segmentation method works better than the benchmark lane marking segmentation methods for lane detection.

Table 4.Comparison of lane marking segmentation methods for Clip \#3

\begin{tabular}{l|l|l|l}
\hline Methods & $\begin{array}{l}\text { Detection } \\
\text { Rate }\end{array}$ & $\begin{array}{l}\text { False } \\
\text { Positive } \\
\text { Rate }\end{array}$ & $\begin{array}{l}\text { False } \\
\text { Negative } \\
\text { Rate }\end{array}$ \\
\hline Proposed & $97.53 \%$ & $2.45 \%$ & $0.02 \%$ \\
FIRSA & $31.94 \%$ & $66.05 \%$ & $2.02 \%$ \\
Canny & $95.86 \%$ & $4.11 \%$ & $0.02 \%$ \\
Sobel & $95.75 \%$ & $4.23 \%$ & $0.02 \%$ \\
Prewitt & $96.71 \%$ & $3.29 \%$ & $0.00 \%$ \\
Roberts & \multicolumn{2}{|l}{} \\
\hline
\end{tabular}

Table Table shows the proposed FIRSA lane marking segmentation method attained $91.20 \%$ of detection rate and $8.80 \%$ of false positive rate in Clip \#4. As seen from Table Table, detection rate from the Canny method is mostly lower than those of the proposed FIRSA, Sobel, Prewitt, and Roberts lane marking segmentation methods. Also, Clip \#4 only brings a slight advantage in favour of Roberts method (only $1.09 \%$ greater than the proposed FIRSA lane marking segmentation method), while in other two scenarios (Clip \#1 and Clip \#3), the proposed FIRSA lane marking segmentation method seems to have better performance than Roberts method. This situation can be related to the agreement on the proposed FIRSA lane marking segmentation method works better than the benchmark lane marking segmentation methods for lane detection. Also, it is noticeable that, as a result of poor illumination during heavy rain weather and unpredictable illumination interference of vehicles, the proposed VBLD framework may perform worse than it does during sunny weather, which is reflected experimentally in Table Table, Figures Fig. 18a, and Fig. 18b of Clip \#4.

Table 5.Comparison of lane marking segmentation methods for Clip \#4

\begin{tabular}{l|l|l|l}
\hline Methods & $\begin{array}{l}\text { Detection } \\
\text { Rate }\end{array}$ & $\begin{array}{l}\text { False } \\
\text { Positive } \\
\text { Rate }\end{array}$ & $\begin{array}{l}\text { False } \\
\text { Negative } \\
\text { Rate }\end{array}$ \\
\hline Proposed & $91.20 \%$ & $8.80 \%$ & $0.00 \%$ \\
FIRSA & $24.87 \%$ & $72.62 \%$ & $2.51 \%$ \\
Canny & $90.49 \%$ & $9.51 \%$ & $0.00 \%$ \\
Sobel & $89.65 \%$ & $10.35 \%$ & $0.00 \%$ \\
Prewitt & $92.29 \%$ & $7.71 \%$ & $0.00 \%$ \\
Roberts & \multicolumn{2}{|l}{} \\
\hline
\end{tabular}

It is concluded that, during rainy weather, the successfulness of the proposed VBLD framework using the proposed FIRSA lane marking segmentation method mainly depends on clarity of the printed lane markings on road surface and sometimes on illumination availability on the road.

Table 6.Comparison of lane detection results

\begin{tabular}{|c|c|c|c|}
\hline Algorithm & $\begin{array}{l}\text { Average } \\
\text { Detection } \\
\text { rate, } \%\end{array}$ & $\begin{array}{l}\text { Average } \\
\text { False } \\
\text { Detection, } \\
\%\end{array}$ & $\begin{array}{l}\text { Missed } \\
\text { detection } \\
\text { rate, } \%\end{array}$ \\
\hline $\begin{array}{l}\text { Sukriti et al. } \\
(2014)[39]\end{array}$ & 99.97 & 2.18 & - \\
\hline $\begin{array}{l}\text { Gaikwad } \\
\text { and } \\
\text { Lokhande } \\
(2015)[40]\end{array}$ & 97.29 & 2.86 & 3.79 \\
\hline $\begin{array}{l}\text { Bhujbal and } \\
\text { Narote } \\
(2015)[41]\end{array}$ & 98.90 & 1.64 & - \\
\hline $\begin{array}{l}\text { Proposed } \\
\text { VBLD }\end{array}$ & 92.84 & 7.16 & 0 \\
\hline
\end{tabular}

Table T compares the lane detections of the existing lane detection algorithms found in the literature with the proposed VBLD framework. Based on this comparison, the straight and curved lanes were considered in [39], [40] for the average detection rate, average false detection rate, and missed detection rate analysis, where the early stage of the development of lane detection algorithms was focused on detecting straight and curved lanes. Due to advances in DAS and awareness of vehicle safety, daytime and night-time driving environments were considered in the recent developments of lane detection algorithms [41]. Although the proposed VBLD framework uses sunny, cloudy, light rain, and heavy rain driving environment groupings in the lane detection and false detection analysis, straight and curved lanes are also considered in road footage Clips \#1-\#4. Based on the comparison in Table T, VBLD uses less computational time per frame at $6.7 \mathrm{~ms}$ than the existing 
lane detection algorithm [39], [40] at $80 \mathrm{~ms}$ and $35 \mathrm{~ms}$, respectively. It can be seen in Table $\mathrm{T}$ that the existing studies of lane detection algorithms in the literature use only a small number of image frames to evaluate their performance, which hardly validates their lane detection performance under varied road conditions. The proposed VBLD used 11,294 frames of road footage for its lane detection analysis with a zero missed detection rate for all Clips.

\section{Conclusions}

In this paper, a vision-based lane detection (VBLD) framework and finite impulse response saturation autothreshold (FIRSA) lane marking segmentation method have been proposed. The vision-based lane detection framework composed of colour space conversion, region of interest, and FIRSA lane marking segmentation method for pre-processing stage. In the detection stage, the Hough transformation and peak detection, reverse Hough transformation, and the drawing of the detected lines in the original image are implemented. Road footage from highway and urban roads in the city of Malacca were collected for evaluating the performance in lane detection. The detection rate and the false positive rate were studied.
Lane detection rates of $96.45 \%, 86.17 \%, 97.53 \%$, and $91.20 \%$ were achieved on Clip \#1, \#2, \#3, and \#4, respectively. The corresponding false positive detection rates were $3.44 \%, 13.83 \%, 2.45 \%$, and $8.80 \%$. Experimental results have shown the evidences of proposed VBLD framework using the proposed FIRSA lane markings segmentation method obtained satisfactory detection rate and false positive rate results compared to benchmark lane markings segmentation methods. The future work includes mainly the coverage of all-weather test environments. As a part of the intelligent transportation system, lane detection performance can be further enhanced with inclusion of tracking element for smoother lane detection results.

\section{Acknowledgment}

The work described in this paper was supported by Multimedia University (MMU) Mini Fund (Grant No. MMUI/180170) and Malaysia Ministry of Higher Education (MOHE) for FRGS FRGS/1/2018/TK03/MMU/02/1.

This is an Open Access article distributed under the terms of the Creative Commons Attribution License

\section{References}

[1] G. Jacobs and A. Aeron-Thomas, "A review of global road accident fatalities," in RoSPA Road Safety Congress, 1999, pp. 1-15.

[2] G. Jacobs, A. A. Thomas, and A. Astrop, "Estimating global road fatalities," London, 2000.

[3] T. Ruxyn, "Death Rates On Malaysian Roads Is 3rd Highest Globally, More Than China And India," 2017. [Online]. Available: https://says.com/my/news/malaysia-s-roads-among-the-world-smost-dangerous-and-deadliest. [Accessed: 05-Apr-2019].

[4] R. Brand, "These Are the World's Most Dangerous Roads Bloomberg," $2017 . \quad$ [Online]. Available: https:/www.bloomberg.com/news/articles/2017-06-19/add-trafficto-the-risks-of-being-in-emerging-markets-chart. [Accessed: 05Apr-2019].

[5] R. Atan, "General Road Accident Statistics in Malaysia - Datasets MAMPU," 2018. [Online]. Available: http://www.data.gov.my/data/en_US/dataset/general-road-accidentstatistics-in-malaysia. [Accessed: 05-Apr-2019].

[6] H. M. N. Al-Madani, "Global road fatality trends' estimations based on country-wise micro level data," Accid. Anal. Prev., vol. 111, no. November 2017, pp. 297-310, 2018.

[7] H. Naci, D. Chisholm, and T. D. Baker, "Distribution of road traffic deaths by road user group: A global comparison," Inj. Prev., vol. 15, no. 1, pp. 55-59, 2009.

[8] WHO, "WHO | Distribution of road traffic deaths by type of road user," $\quad 2016 . \quad$ [Online]. Available: http://www.who.int/gho/road_safety/mortality/traffic_deaths_distri bution/en/. [Accessed: 23-Jul-2018].

[9] L. Wu, S. Geedipally, and A. M. Pike, "Safety Evaluation of Alternative Audible Lane Departure Warning Treatments in Reducing Traffic Crashes: An Empirical Bayes Observational Before-After Study," Transp. Res. Rec. J. Transp. Res. Board, p. 036119811877648 , Jun. 2018.

[10] T. L. Brown, J. Gaspar, D. Marshall, and J. D. Lee, "How does lane departure warning effectiveness vary by severity of departure," in Proceedings of the Human Factors and Ergonomics Society, 2017, vol. 2017-Octob, no. 2008, pp. 1929-1933.

[11]G. M. Fitch, S. E. Lee, S. Klauer, J. Hankey, J. Sudweeks, and T. Dingus, "Analysis of Lane-Change Crashes and Near-Crashes," 2009.

[12]E. Kopits and M. Cropper, "Traffic fatalities and economic growth," Accid. Anal. Prev., vol. 37, no. 1, pp. 169-178, 2005.

[13]F. Wegman, "The future of road safety: A worldwide perspective," IATSS Res., vol. 40, no. 2, pp. 66-71, 2017.
[14]H. Yoshida, M. Omae, and T. Wada, "Toward next active safety technology of intelligent vehicle," J. Robot. Mechatronics, vol. 27, no. 6 , pp. 610-616, 2015.

[15]M. Haloi and D. B. Jayagopi, "A robust lane detection and departure warning system," in Intelligent Vehicles Symposium (IV), 2015 IEEE, 2015, pp. 126-131.

[16] S. P. Narote, P. N. Bhujbal, A. S. Narote, and D. M. Dhane, "A review of recent advances in lane detection and departure warning system," Pattern Recognit., vol. 73, pp. 216-234, 2018.

[17]P. Viswanath et al., "A diverse low cost high performance platform for advanced driver assistance system (ADAS) applications," in IEEE Computer Society Conference on Computer Vision and Pattern Recognition Workshops, 2016, pp. 819-827.

[18]C. R. Jung and C. R. Kelber, "A robust linear-parabolic model for lane following," in Computer Graphics and Image Processing, 2004. Proceedings. 17th Brazilian Symposium on, 2004, pp. 72-79.

[19] C. R. Jung and C. R. Kelber, "An improved linear-parabolic model for lane following and curve detection," in Computer Graphics and Image Processing, 2005. SIBGRAPI 2005. 18th Brazilian Symposium on, 2005, pp. 131-138.

[20]F. Meng and C. Spence, "Tactile warning signals for in-vehicle systems," Accid. Anal. Prev., vol. 75, pp. 333-346, 2015.

[21] S. Vacek, C. Schimmel, and R. Dillmann, "Road-marking analysis for autonomous vehicle guidance," in EMCR, 2007, pp. 1-6.

[22]Logitech, "Logitech C525 HD Webcam," Logitech, 2018. [Online]. Available: https://www.logitech.com/en-us/product/hd-webcamc525. [Accessed: 03-Dec-2018].

[23] S. Sternlund, J. Strandroth, M. Rizzi, A. Lie, and C. Tingvall, "The effectiveness of lane departure warning systems-A reduction in real-world passenger car injury crashes," Traffic Inj. Prev., vol. 18, no. 2, pp. 225-229, 2017.

[24]A. Sudhakaran, "Lateral control using a MobilEye camera for lane keeping assist," Eindhoven University of Technology, 2017.

[25]P. Chandrasekar and J. Y. Ryu, "Design of programmable digital FIR/IIR filter with excellent noise cancellation," Int. J. Appl. Eng. Res., vol. 11, no. 15, pp. 8467-8470, 2016.

[26] M. Shah, Fundamental of Computer Vision. Orlando, FL, 1997.

[27]T. J. Terrell and R. J. Simpson, "Two-dimensional FIR filter for digital image processing," J. Inst. Electron. Radio Eng., vol. 56, no. 3, pp. 103-106, 1986.

[28]N. Otsu, "A Threshold Selection Method from Gray-Level Histograms," IEEE Trans. Syst. Man. Cybern., vol. 9, no. 1, pp. 62$66,1979$. 
[29]H. J. Vala and P. A. Baxi, "2013-a-Review-on-Otsu-ImageSegmentation-Algorihm," Int. J. Adv. Res. Comput. Eng. Technol., vol. 2, no. 2, pp. 387-389, 2013.

[30] S. Bangare and S. Patil, "Reviewing Otsu' s Method For Image Thresholding," Int. J. Appl. Eng. Res., vol. 10, no. 9, pp. $21777-$ 21784, 2015.

[31] W. Wei and H. Xinming, "An FPGA co-processor for adaptive lane departure warning system," in Circuits and Systems (ISCAS), 2013 IEEE International Symposium on, 2013, pp. 1380-1383.

[32] C. Mu and X. Ma, "Lane detection based on object segmentation and piecewise fitting," TELKOMNIKA Indones. J. Electr. Eng., vol. 12, no. 5, pp. 3491-3500, 2014.

[33]R. O. Duda and P. E. Hart, "Use of the hough transformation to detect lines and curves in pictures," Commun. ACM, vol. 15, no. 1, pp. $11-15,1972$.

[34] Autoworld, "Specifications for Perodua Kancil 660 EX,' Autoworld, 2018. [Online]. Available: http://autoworld.com.my/news/2002/09/28/Specifications-forKancil-660-EX-850-EX-and-850-EZi/. [Accessed: 03-Dec-2018].

[35] J. Canny, "A computational approach to edge detection.," IEEE Trans. Pattern Anal. Mach. Intell., vol. 8, no. 6, pp. 679-698, 1986.

[36]S. Gupta and S. Ghosh Mazumdar, "Sobel edge detection algorithm," Int. J. Comput. Sci. Manag. Res., vol. 2, no. 2, pp. 1578-1583, 2013.

[37] Gonzales, R. C., and R. E. Woods, Digital Image Processing, 2nd ed. Englewood Cliffs, NJ: Prentice-Hall, 2002.

[38]M. Kumar and R. Saxena, "Algorithm and technique on various edge detection: a survey," Signal Image Process. An Int. J., vol. 4, no. 3, pp. 65-75, 2013.

[39] S. Sukriti, L. Manisha, and S. Ritika, "Improved lane detection using hybrid median filter and modified hough transform," Int. J. Adv. Res. Comput. Sci. Softw. Eng., vol. 4, no. 1, pp. 30-37, 2014.

[40] V. Gaikwad and S. Lokhande, "Lane departure identification for advanced driver assistance," IEEE Trans. Intell. Transp. Syst., vol. 16, no. 2, pp. 910-918, 2015.

[41]P. N. Bhujbal and S. P. Narote, "Lane departure warning system based on Hough transform and Euclidean distance," in Proceedings of 2015 3rd International Conference on Image Information Processing, ICIIP 2015, 2015, pp. 370-373. 